\title{
Disorder screening near the Mott-Anderson transition
}

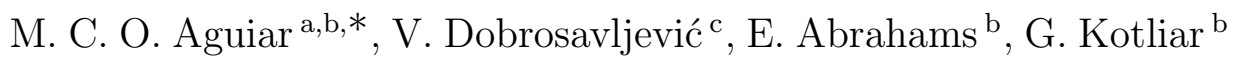 \\ ${ }^{a}$ Departamento de Física, Universidade Federal de Minas Gerais, Av. Antônio Carlos, 6627, Belo Horizonte, MG, Brazil \\ Center for Materials Theory, Serin Physics Laboratory, Rutgers University, 136 Frelinghuysen Road, Piscataway, New Jersey 08854, USA \\ ${ }^{\mathrm{c}}$ Department of Physics and National High Magnetic Field Laboratory, Florida State University, Tallahassee, FL 32306, USA
}

\section{Abstract}

Correlation-driven screening of disorder is studied within the typical-medium dynamical mean-field theory (TMT-DMFT) of the Mott-Anderson transition. In the strongly correlated regime, the site energies $\varepsilon_{R}^{i}$ characterizing the effective disorder potential are 'strongly renormalized due to the phenomenon of Kondo pinning. This effect produces very strong screening when the interaction (4) $U$ is stronger then disorder $W$, but applies only to a fraction of the sites in the opposite limit $(U<W)$.

Introduction - Theories that are able to capture both the Mott [1] and the Anderson [2] mechanisms for electron localization have remained elusive despite many years of effort. An attractive approach to this difficult problem has recently been proposed by combining the dynamical meanfield theory (DMFT) 3 of the Mott transition, and the typical medium theory (TMT) [4] of Anderson localization. This new formulation of the Mott-Anderson problem has been explored in recent work by Vollhardt and collaborators [5] using numerical renormalization-group methods, but the precise mechanism for the critical behavior of this model remains to be elucidated. Here we examine the mechanism for disorder screening within this theory, which explains several aspects of the results found in Ref. [5]

Within TMT-DMFT, a lattice problem is mapped onto an ensemble of single-impurities problems, which are embedded in a self-consistently determined bath. Recent work of Ref. 6] examined the behavior of a collection of singleimpurity models in the situation where the bath seen by the impurities was chosen to mimic the approach to the MottAnderson transition. In this work, the impurity quasiparticle weight $Z_{i}$ was shown to present a scaling behavior as a function of the on-site energy $\varepsilon_{i}$ and the distance $t$ to the transition. These findings, however, are not sufficient to address the disorder screening behavior of the model, which requires the description of the renormalized disor-

\footnotetext{
* Corresponding author. Tel: +55 31 3499-5671 fax: +55 31 34995600

Email address: aguiar@fisica.ufmg.br (M. C. O. Aguiar).
}

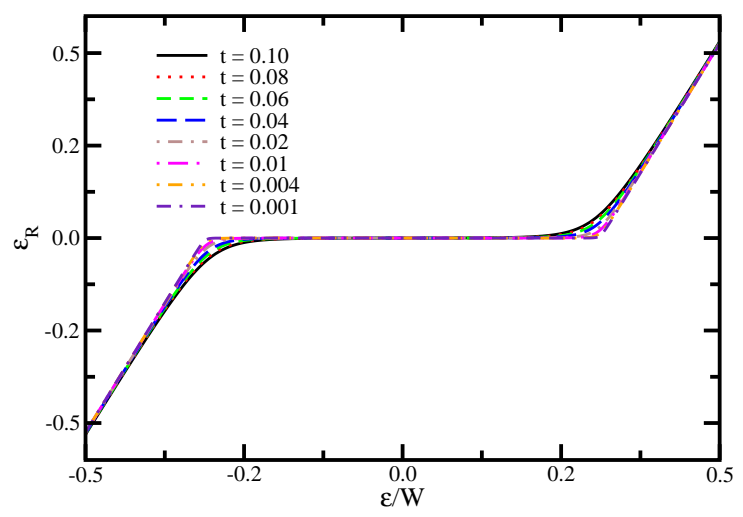

Fig. 1. Renormalized energy $\varepsilon_{R}$ as a function of the on-site energy $\varepsilon$ for a collection of single-impurity problems close to the Mott-Anderson transition $(t \rightarrow 0)$. The parameters used were $U=1.75$ and $W=2.8$.

der potential. In this paper, we demonstrate that a scaling procedure similar to that presented in Ref. 6 can also be carried on for the renormalized energy $\varepsilon_{R}^{i}$.

Renormalization of the disorder potential - We consider a collection of Anderson impurity models [6] with on-site repulsion $U$, on-site energies $\varepsilon_{i}$, and the total spectral weight $t$ of the cavity field. Without loss of generality [6, we consider a featureless model bath with non-vanishing spectral weight for $-t / 2<\omega<t / 2$ and zero otherwise. Our goal is to describe the statistics of the renormalized site energies as the metal-insulator transition is approached, corresponding to $t \rightarrow 0$ within the TMT-DMFT scheme. 


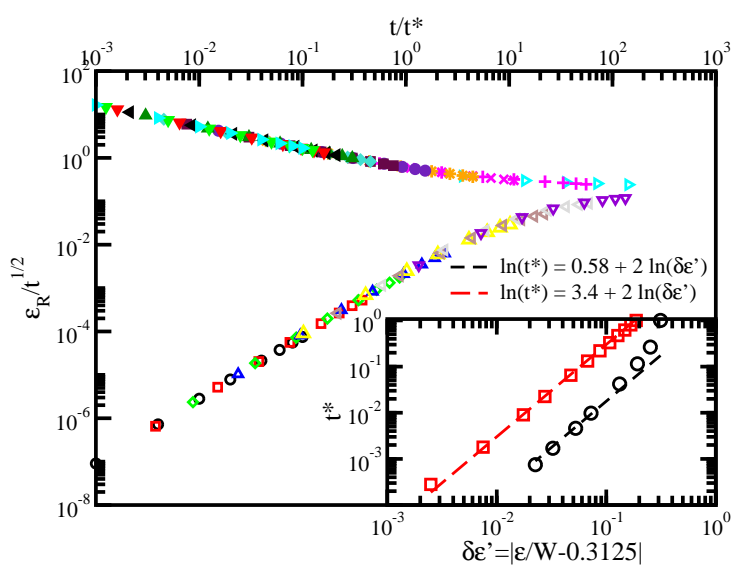

Fig. 2. Scaled renormalized energy $\varepsilon_{R} / t^{1 / 2}$ as a function of $t / t^{*}(\delta \varepsilon)$ showing that the results for different (and positive) $\varepsilon$ can be collapsed onto a single scaling function with two branches. Different symbols correspond to different $\varepsilon$; the upper (bottom) branch contains results for $\varepsilon>U / 2(\varepsilon<U / 2)$. The inset shows the scaling parameter $t^{*}$ as a function of $|\varepsilon / W-0.3125|$ for the upper (squares) and bottom (circles) branches. The parameters used were $U=1.75$ and $W=2.8$.

The impurity models were solved at zero temperature using the SB4 method 76], which provides a parametrization of the low-energy (quasiparticle) part of the local Green's function, given by

$$
G_{i}\left(\omega_{n}\right)=\frac{Z_{i}}{i \omega_{n}-\varepsilon_{R}^{i}-Z_{i} \Delta\left(\omega_{n}\right)} .
$$

Here $Z_{i}$ is the local quasiparticle weight and $\varepsilon_{R}^{i}$ is the renormalized site energy. The details of the calculations mirror those of Ref. 6.

The results for the renormalized energy $\varepsilon_{R}^{i}$ as a function of $-W / 2<\varepsilon_{i}<W / 2$, in the vicinity of the MottAnderson transition $(t \rightarrow 0)$, are shown in Fig. 1, As in Ref. [6], we find two-fluid behavior, where sites with $\left|\varepsilon_{i}\right|<$ $U / 2$ turn into local magnetic moments, corresponding to "Kondo pinning" [ 8$] \varepsilon_{R}^{i} \rightarrow 0$. For the remaining sites, $\varepsilon_{R}^{i} \rightarrow$ $\varepsilon_{i}+U / 2$ or $\varepsilon_{R}^{i} \rightarrow \varepsilon_{i}-U / 2$, as they become, respectively, doubly occupied (those with $\varepsilon_{i}<-U / 2$ ) or empty (those with $\varepsilon_{i}>U / 2$ ). We should emphasize that such two-fluid behavior thus emerges only for sufficiently strong disorder, such that $U<W$. Otherwise all sites turn into local magnetic moments, and the Mott transition for moderate disorder retains a character similar to that found within the standard DMFT approach [8].

Scaling analysis - These results can alternatively be presented in a scaling form, as shown in Fig. 2. Here, we show that it is possible to collapse the family of curves $\varepsilon_{R}(t, \delta \varepsilon) / t^{0.5}$, where $\delta \varepsilon=\left(\varepsilon_{i}-\varepsilon^{*}\right) / \varepsilon^{*}$ and $\varepsilon^{*}=U / 2$, onto a single universal scaling function $\varepsilon_{R}(t, \delta \varepsilon) / t^{0.5}=$ $f\left[t / t^{*}(\delta \varepsilon)\right]$ with two branches, one for $\varepsilon_{i}<\varepsilon^{*}$ and other for $\varepsilon_{i}>\varepsilon^{*}$. In agreement with Ref. [6] (inset of Fig. 2), the crossover scale $t^{*}(\delta \varepsilon) \sim|\delta \varepsilon|^{\phi}$, with exponent $\phi=2$. In the limit $t \rightarrow 0$, we find that the branch corresponding to $\varepsilon_{i}<\varepsilon^{*}$ has the asymptotic form $f(x) \sim x^{3 / 2}$ (here $x=$ $\left.t / t^{*}(\delta \varepsilon)\right)$, corresponding to $\varepsilon_{R}(t) \sim t^{2}$. Similarly, for $\varepsilon_{i}>$ $\varepsilon^{*}, f(x) \sim x^{-1 / 2}$ corresponding to $\varepsilon_{R}(t) \sim$ constant. For

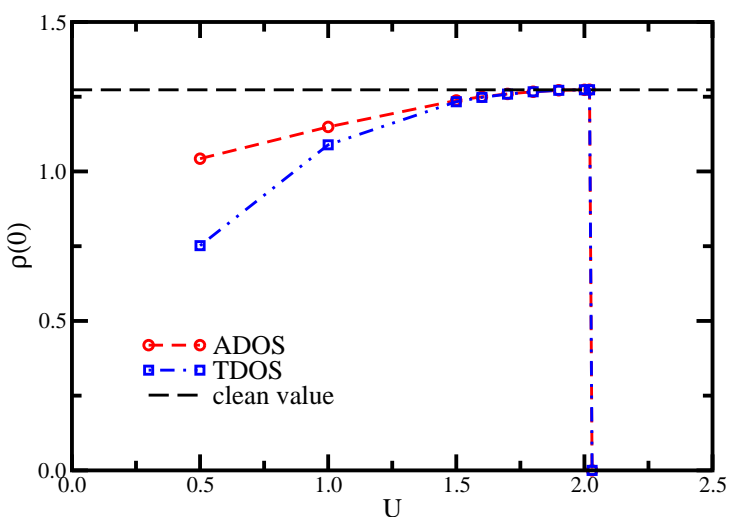

Fig. 3. Arithmetic and geometric density-of-states (ADOS and TDOS, respectively) at the Fermi level as a function of $U$, for $W=1.5$, when the TMT-DMFT self-consistent loop is performed.

$x \gg 1$ the two branches merge, viz. $f(x) \sim A \pm B^{ \pm} x^{-0.5}$.

Disorder screening - Within TMT-DMFT, the Anderson localization effects are manifested by the reduction of the typical density of states (TDOS), since the (algebraic) average (ADOS) remains finite even in an Anderson insulator. When disorder is strongly screened due to the correlation effects, the two quantities should not differ much, as illustrated by the results of Fig. 3. Here we present the results of the fully self-consistent solution, as the Mott-like transition is approached by increasing $U$ for $W=1.5$. Close to the transition, both averages approach the clean limit (dashed line), indicating a strong screening effect. These results are consistent with those found in the numerical renormalization group solution of the TMT-DMFT equation of Ref. [5].

As discussed above, strong disorder screening is expected near the Mott-like transition $(U>W)$, which indeed corresponds to the mechanism responsible for the results in Fig. 3. When the transition is approached at strong disorder $(U<W)$ (not shown), strong screening effects are found only for a fractions of the sites (i.e of the volume of the sample), indicating different critical behavior at the MottAnderson transition. The details of the critical behavior in this case will be discussed elsewhere.

Acknowledgements - This work was partially supported by NSF grants DMR-0312495 (M.C.O.A.), DMR-0234215 and DMR-0542026 (V.D.) and DMR-0096462 (G.K.).

\section{References}

[1] N.F. Mott, Metal-insulator Transitions (Taylor and Francis, London, 1974).

[2] P.W. Anderson, Phys. Rev. 109 (1958) 1498.

[3] A. Georges et al., Rev. Mod. Phys. 68 (1996) 13.

[4] V. Dobrosavljević et al., Europhys. Lett. 62 (2003) 76.

[5] K. Byczuk, W. Hofstetter, and D. Vollhardt, Phys. Rev. Lett. 94 (2005) 056404.

[6] M.C.O. Aguiar et al., Phys. Rev. B 73 (2006) 115117.

[7] G. Kotliar and A.E. Ruckenstein, Phys. Rev. Lett. 57 (1986) 1362.

[8] D. Tanasković et al., Phys. Rev. Lett. 91 (2003) 066603. 